\title{
Statement about ownership and other particulars of
}

Resonance - journal of science education

1. Place of Publication :

Bengaluru

2. Periodicity of Publication :

Monthly

3. Printer's Name :

Amitabh Joshi

Indian Academy of Sciences

Bengaluru 560080

4. and 5. Publisher and Editor :

Amitabh Joshi

6. Nationality:

Indian

7. Address :

Indian Academy of Sciences

P.B. No. 8005, Bengaluru 560080

8. Name and Address of the Owner :

Indian Academy of Sciences

Bengaluru 560080

I, Amitabh Joshi, hereby declare that the particulars given above are true to the best of my knowledge.

Date: 1st March 2018

Amitabh Joshi

Signature of Publisher 